Artículos Científicos

\title{
La actividad agrícola en localidades rurales en procesos conurbatorios: Una aproximación mediante el diagrama de Ishikawa
}

Agricultural Activity in Rural Localities in Conurbation Processes: An Approximation Using the Ishikawa Diagram

Atividade agrícola em cidades rurais em processos de conurbação: uma abordagem usando o diagrama de Ishikawa

José Luis Carmona Silva

Benemérita Universidad Autónoma de Puebla, Puebla, México jlcarmonas@yahoo.com.mx https://orcid.org/0000-0002-0858-2792

José Aurelio Cruz de los Ángeles Benemérita Universidad Autónoma de Puebla, Puebla, México pepejac52@yahoo.com.mx https://orcid.org/0000-0002-8682-366X

Lizbeth Sánchez Flores Benemérita Universidad Autónoma de Puebla, Puebla, México lisanflo@yahoo.com.mx https://orcid.org/0000-0003-1318-3873 


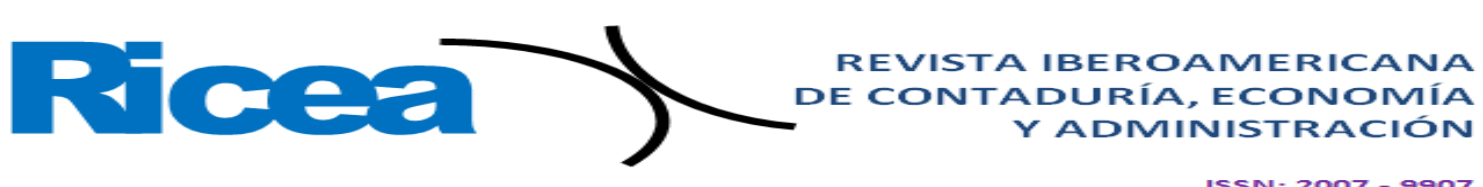

\section{Resumen}

El objetivo del presente trabajo fue analizar los cambios que sufre la actividad agrícola en una localidad rural que se encuentra bajo un proceso conurbatorio. Esto mediante una metodología innovadora que empleó herramientas como el diagrama de Ishikawa y guías de entrevistas, las cuales sirvieron para reforzar y reafirmar el dato numérico de los resultados obtenidos. Santa María Texcalac, Tlaxcala, es una localidad que presenta estas características. Su actividad económica principal, la agricultura, ha sufrido cambios significativos a consecuencia de este tipo de procesos. Para obtener esta información se empleó una metodología mixta: se aplicaron 85 cuestionarios a una muestra representativa, se realizaron 6 entrevistas a profundidad con informantes clave y se realizó un taller de Ishikawa con personajes de la localidad. Los resultados reflejan una actividad agrícola en peligro de extinción, una pérdida significativa de superficie para cultivo por cambio de uso de suelo, un reclamo cada vez más débil de apoyo al campo y cada vez más fuerte de creación de empleos, un desaliento para seguir siendo campesinos y una búsqueda de otra actividad económica para los hijos.

Palabras clave: actividad agrícola, localidad rural, proceso conurbatorio.

\section{Abstract}

The objective of this work was to analyze the changes that agricultural activity undergoes in a rural town that is under a conurbation process. This through an innovative methodology that used tools such as the Ishikawa diagram and interview guides, which served to reinforce and reaffirm the numerical data of the results obtained. Santa María Texcalac, Tlaxcala, is a town that has these characteristics. Its main economic activity, agriculture, has undergone significant changes as a result of this type of process. To obtain this information, a mixed methodology was used, applying 85 questionnaires to a representative sample, six in-depth interviews were carried out with key informants and an Ishikawa workshop was held with local characters. The results reflect an agricultural activity in danger of extinction, a significant loss of area for cultivation due to a change in land use, an increasingly weak demand for support to the countryside and an increasingly strong job creation, a discouragement to remain peasants and a search for another economic activity for the children. 


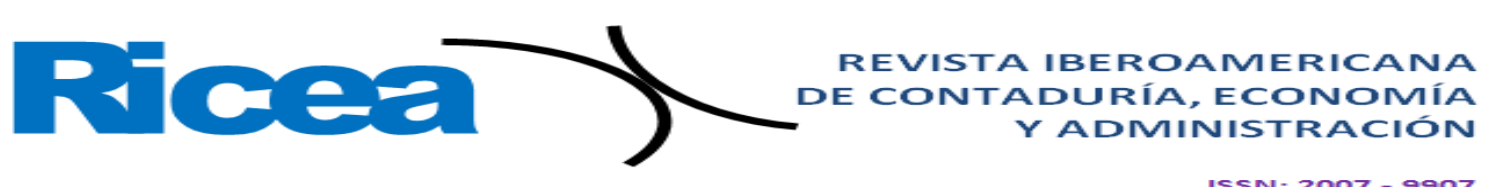

El proceso mediante el cual se lleva a cabo el crecimiento sin control y sin planes de las urbes es actualmente tan cotidiano que no se percibe, pareciera algo muy lógico y natural no solo en nuestro país sino en otras latitudes: así sucede en Centroamérica (Valladares, 2008), Sudamérica (Moreno, 2004) o en Europa (Naredo, 2003). De hecho, según cifras proporcionadas por la Organización de las Naciones Unidas (ONU), para el año 2030 más de dos terceras partes de la población mundial vivirá en zonas urbanas (Fondo de Población de las Naciones Unidas [FNUAP], 2018).

Las conurbaciones, sumadas a otras variables, evidencian una veloz e incontrolable concentración de la población en las metrópolis de más de 500000 habitantes. Esta concentración no controlada causa desempleo, subempleo, problemas de seguridad, problemas de uso de suelo, carencia de servicios públicos, pugnas administrativas y graves problemas ambientales, entre otros (Naredo, 2000).

Los anquilosados y estáticos modelos de uso de suelo y las actividades agropecuarias de las localidades rurales remitían a un modelo organizado en círculos concéntricos alrededor de un foco único que era la ciudad. Definitivamente este modelo ya no tiene validez. Si quisiéramos entender y encasillar en categorías mentales del siglo pasado a los procesos conurbatorios de hoy en día pronto caeríamos en la cuenta de que no existe correspondencia: el modelo de conurbación que impera en nuestros días toma como punto de partida las vías de comunicación (Comisión Económica para América Latina y el Caribe [Cepal], 2010).

Una ciudad, bajo este modelo, no necesariamente conurba a la localidad rural más próxima, sino que la tendencia es conurbar a las localidades rurales cercanas a las vías de comunicación que unen a las ciudades más importantes. En el estado de Tlaxcala es fácil observar el proceso conurbatorio que está en marcha en la carretera que va de Apizaco a Huamantla. A los lados de esta carretera han proliferado micro, pequeñas y medianas empresas (mipymes) y zonas habitacionales a tal grado que ya son pocos los tramos en los que no hay construcciones. Lo mismo sucede en la carretera federal que une las ciudades de Puebla y Tlaxcala. Por lo que a estas alturas se puede afirmar la existencia de una verdadera conurbación fomentada y basada más por la vía de comunicación que por la cercanía física de las poblaciones que se conurban.

Estos ejemplos citados no son únicos ni excepcionales, sino que representan el común denominador del esquema o modelo de los procesos actuales de conurbación. Las características propias de este modelo son ejemplificadas también en otras latitudes, como la 


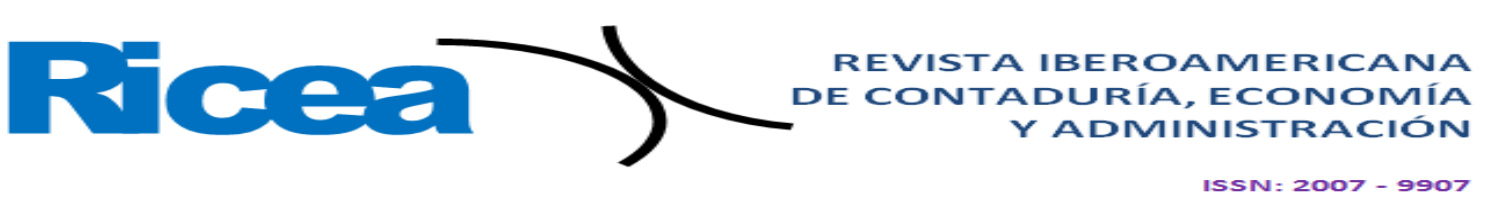

mencionada por Navarro (2005). Si bien este modelo es el ahora predominante, como lo demuestra la evidencia empírica, ya desde la década de los 60 fue descrito como una de las formas de la evolución de las ciudades (Moreno, 2004).

No debe escapar la consideración de la compleja problemática que estos procesos conllevan, sobre todo si consideramos de manera específica la incorporación económica y territorial de comunidades periféricas eminentemente rurales a la dinámica de la urbe. El proceso de conurbación que sufren algunas localidades rurales, cuya principal actividad económica es o era la agricultura, ocasiona una serie de cambios profundos que trastoca de manera integral a dichas comunidades, mutando su panorama físico, sus actividades económicas y hasta su comportamiento sociocultural.

En un proceso conurbatorio en el que se involucra una mancha urbana y una localidad rural uno de los factores que sufre los cambios más profundos es el uso del suelo. Los dueños de los terrenos no ambicionan buscar alternativas que hagan más productivas sus propiedades; su intención y ambición es venderlos al mejor postor.

La actitud de los campesinos hacia su "campo" es diametralmente opuesta a la que tenían en un pasado muy próximo: no hace mucho defendían su actividad agrícola con pasión y coraje, la presumían a la menor oportunidad, recreaban la vista con sus terrenos cuando su siembra estaba en pleno verdor. Sin embargo, ahora se rigen en términos de cuánto dinero podrán obtener por su venta. Algunos autores sostienen que ante el crecimiento de la ciudad, la tierra se ha convertido poco a poco más en un espacio para construir que en uno de cultivo agrícola (Kautsky, 2002).

El problema de esta investigación consiste en conocer con precisión si en un marco social, económico y geográfico que caracteriza a las comunidades rurales en proceso de conurbación, como es el caso de la localidad de Santa María Texcalac, Tlaxcala, la actividad agrícola se encuentra marginada y desestructurada, con poca importancia económica sepultada por la influencia de los procesos urbanos; o por el contrario, es una actividad que ha encontrado mecanismos de adaptación no solo para sobrevivir, sino para dinamizarse aprovechando los nuevos elementos citadinos que su contexto le ofrece.

Más allá de este conocimiento, el proyecto intenta interiorizar en los mecanismos y causalidades que los propios habitantes de Sta. María Texcalac identifican como generadores de los fenómenos situacionales presentes. 


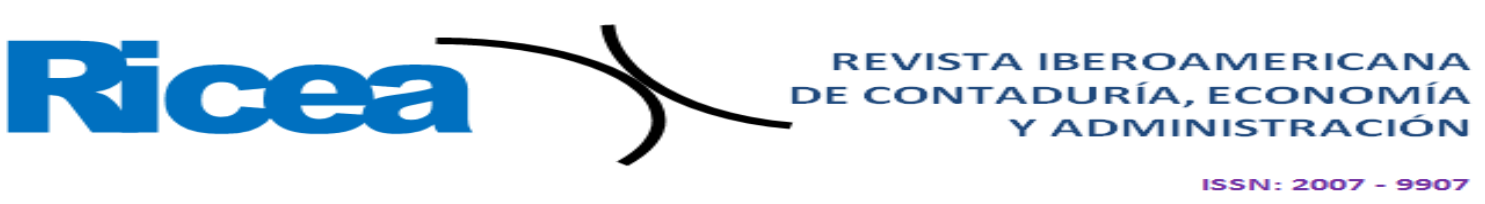

a primera vista. Para cualquier observador es obvio que la parte sur de la localidad es la que está sufriendo una invasión de industrias y centros de trabajo equivalentes a talleres de toda índole. Esto es explicable por ser la parte de la población que es atravesada por la carretera federal México-Veracruz. La parte central de la población puede decirse que es la que presenta una panorámica más urbana, sin alcanzar totalmente las cualidades propias de una urbe. Por otra parte, la zona norte es la que posee rasgos más cercanos a la ruralidad, no solo por los campos dedicados todavía a la agricultura, sino por las características de las viviendas, caminos y veredas, entre otros.

Como ya se mencionó, la población objetivo fue la comunidad de Santa María Texcalac, y se tomó como base la información obtenida del XII Censo General de Población y Vivienda (Inegi, 2010). Esta localidad cuenta con una población total de 5284 habitantes, los cuales forman 1096 familias, con un promedio de ocupantes por vivienda de 4.82. En consecuencia, la muestra se obtuvo de este universo de familias. Para realizar este cálculo se recurrió al método de muestreo considerando la varianza máxima. Las características que utilizamos fueron que las familias se dedicaran o no a la actividad agrícola en esta localidad. Para seleccionar el tamaño de muestra se tomó una precisión de 10 \% y una confiabilidad de $95 \%$. Se obtuvo un resultado de 88 encuestas a realizar.

No se consideró otro método de muestreo debido a que no se contó con estratos o subgrupos en los que se pudiera dividir a la población. Se sabe, por un primer recorrido, que la actividad agrícola se sigue practicando, aunque es evidente que no tiene el mismo peso que años atrás, como la misma gente de la localidad indica; y de la misma forma encontramos diferentes usos del suelo.

\section{Diseño y aplicación de un cuestionario a la muestra seleccionada}

El cuestionario siempre ha sido, en los diferentes diseños de investigación, una herramienta valiosa para obtener información. Para Hernández, Fernández y Baptista (2014) el cuestionario "consiste en un conjunto de preguntas respecto a una o más variables a medir" (p. 217). El propósito de elaborar un cuestionario se ve todavía mejor reflejado en la descripción que consigna Quispe (1999: 125): "Es un formato de preguntas debidamente ordenadas y organizadas para obtener la información que se requiere y permita su sistematización, tabulación y posterior interpretación”. Por la elasticidad y los múltiples temas que se pueden manejar en este tipo de formato, permite obtener información sobre qué 


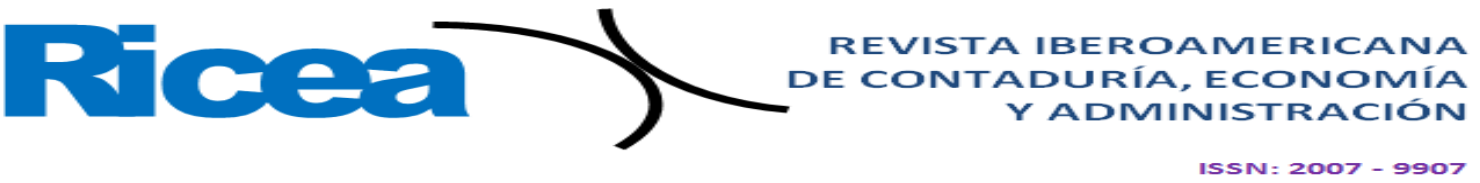

es lo que hace la gente, qué es lo que tiene, qué es lo que piensa, conoce, siente o desea. Precisamente estas son las áreas hacia las que hemos dirigido el interrogatorio, siempre con el tema de la agricultura como eje rector.

El agricultor como fuente primaria de información es totalmente indiscutible. Si alguien conoce un proceso es quien vive cotidianamente inmerso en él. Es el agricultor el que se beneficia o el que sufre las consecuencias de un proceso de conurbación como el que se da actualmente en Sta. María Texcalac.

\section{Entrevista con autoridades e informantes claves de la localidad}

El objetivo de esta investigación, dadas sus características, es de alguna manera fenomenológico. De ahí que una parte importante de la metodología usada deba corresponder a esta perspectiva teórica. Bajo esta óptica, la entrevista se convierte en una herramienta valiosa tanto por la cantidad como por la calidad de información que produce. La entrevista, sobre todo, permite tener una visión de la temática desde la propia perspectiva del protagonista; es un instrumento que ayuda a limpiar de prejuicios el acervo de información que el investigador va recopilando, analizando e intentando interpretar desde una posición externa.

Por otra parte, el taller de causa-efecto responde apropiadamente a las exigencias de la observación participante (Bracamonte, 2015); y las entrevistas a las autoridades y personajes de la localidad responden a las entrevistas de profundidad. Tanto estas como la observación son consideradas como métodos cualitativos de investigación.

En el caso de esta investigación se eligió el sistema de entrevista no dirigida, en la que, una vez planteado el tema, se le da al entrevistado amplia libertad de expresión — aunque se cuida de una manera sutil que no divague por mucho tiempo en otros temas ajenos al interés de la investigación. Tocante al tema de los entrevistados, precisamente, un punto que se cuidó con sumo esmero es el siguiente: que la elección recayera en personas con un amplio conocimiento de la localidad y que, de alguna manera, hubieran estado involucrados en la detección y solución de problemas comunitarios. Se buscaron personas con amplia experiencia en los asuntos locales y con aceptación social dentro de la comunidad.

En sintonía con lo anterior, dos de los seleccionados han ocupado los tres puestos de autoridad máxima que existe dentro de la localidad: presidente de comunidad, comisariado ejidal y fiscal. Uno de los entrevistados se encuentra entre las dos personas más antiguas de 


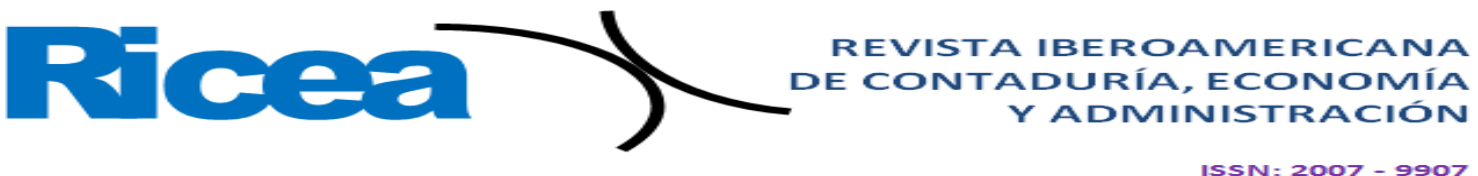

la localidad (95 años); pese a su edad, sus capacidades motrices, intelectuales y emocionales se encuentran en un alto grado de aceptabilidad. En general, todos los entrevistados han ocupado algún puesto de autoridad local.

\section{Realización de un taller con técnica Ishikawa \\ Diagrama causa-efecto}

Es obvio que los diagramas son una herramienta útil y efectiva para representar visualmente un fenómeno determinado, numérico o no. Por ello, la utilización de estos es muy socorrida en casi todos los diseños de experimentación y tácticas de investigación en todos los campos de la ciencia. Para cada campo o para cada problemática analizada, es casi seguro que existe un tipo de diagrama adecuado que, utilizado con propiedad, proporciona ayuda invaluable para la comprensión y entendimiento de los múltiples vértices que por lo general presenta todo fenómeno lo suficientemente interesante para ser investigado.

Las ciencias en su multiplicidad han adoptado de alguna manera los diagramas que más se acomodan al análisis de su propia fenomenología. En esa tónica, es difícil, por ejemplo, que los sistemas de producción en busca de mejorar sus productos mediante el control de calidad no usen el diagrama de Pareto. De la misma manera, un diseño de investigación que tiene por objeto la detección de factores multicausales de una realidad presente, como es el caso de nuestro estudio, debe preocuparse por incluir dentro de su estrategia metodológica un diagrama, al menos, que enriquezca sustancialmente el acopio de información con el que contará el investigador para lograr los objetivos propuestos.

Dentro de los diagramas para el descubrimiento de los factores policausales de una situación dada nos inclinamos por elegir el diagrama de causa-efecto, comúnmente referido por el apellido de su creador: Ishikawa, y, aunque menos frecuente, también denominado diagrama de espina de pescado. Este diagrama se ha hecho acreedor de tal mote por la forma que adquiere su representación. Algunas de sus ventajas son las siguientes:

- $\quad$ Se obtiene mucha información en poco tiempo.

- La información se limpia por la argumentación de los participantes.

- $\quad$ Existe retroalimentación instantánea.

- Los participantes son protagonistas de la "situación” analizada.

- $\quad$ La riqueza de información puede arrojar un insight inesperado. 


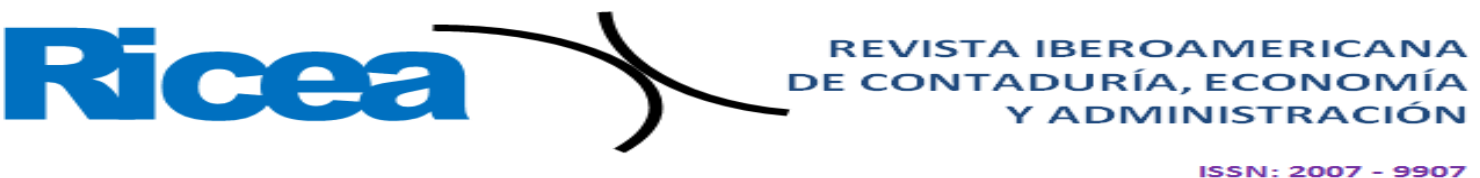

- La interrelación de los participantes funciona como estímulo positivo para la participación heterogénea.

Mientras que las principales desventajas son:

- $\quad$ No se puede evaluar numéricamente.

- $\quad$ Sus resultados son estrictamente cualitativos.

- $\quad$ Permite solo la deducción lógica pero no matemática.

- $\quad$ Si no existe un moderador hábil, la opinión puede focalizarse en un líder.

\section{Mecánica del diagrama de causa-efecto}

En términos muy generales diremos que el diagrama de causa-efecto se utiliza para ordenar y representar gráficamente las opiniones o posturas que resultan de un proceso de lluvia de ideas que emite cada uno de los miembros de un grupo al dar respuesta a una pregunta que se plantea como inicio o apertura del análisis.

De lo anterior se deduce que primero se seleccionó un equipo de trabajo o grupo de expertos o conjunto de personas involucradas en el tema. Se eligió una pregunta que tocaba directamente el tema de análisis y bajo la observación de un moderador, que tuvo como función impedir el acaparamiento de respuestas y motivar la participación equilibrada. Se dejó fluir libremente la lluvia de ideas que cada uno de los componentes del grupo externó. Las ideas obtenidas fueron registradas mediante un apuntador y a través de una grabación para que posteriormente se estratificaran y clasificaran haciéndolas aptas para ser representadas en un diagrama de Ishikawa.

La elaboración del diagrama causal proporcionó la oportunidad de retomar bajo muchas perspectivas el problema o tema analizado, ya que la multiplicidad de ideas externadas permitió que fueran categorizadas en la cantidad de apartados que la lluvia de ideas exigió.

Los pasos que la mecánica propone son:

1) Escribir el tema o problema seleccionado en un recuadro colocado a la mitad en el lado derecho de una hoja y dibujar una flecha gruesa que comienza en el lado izquierdo y apunta hacia el recuadro (ver figura 1). 
Figura 1. Tema seleccionado

Tema o problema seleccionado

Fuente: Elaboración propia con información de Cantú (2011)

2) Categorizar la lluvia de ideas. Titular apropiadamente cada categoría o clasificación y escribir este título en flechas secundarias que apuntan al cuerpo de la flecha dibujada (ver figura 2).

3) Escribir con mayor detalle los elementos pertenecientes a cada una de las categorías, colocando a estos elementos a los lados de las flechas secundarias, según sea la categoría o la que pertenezca al factor seleccionado por su tasa de aparición (ver figura 3).

Figura 2. Categoría

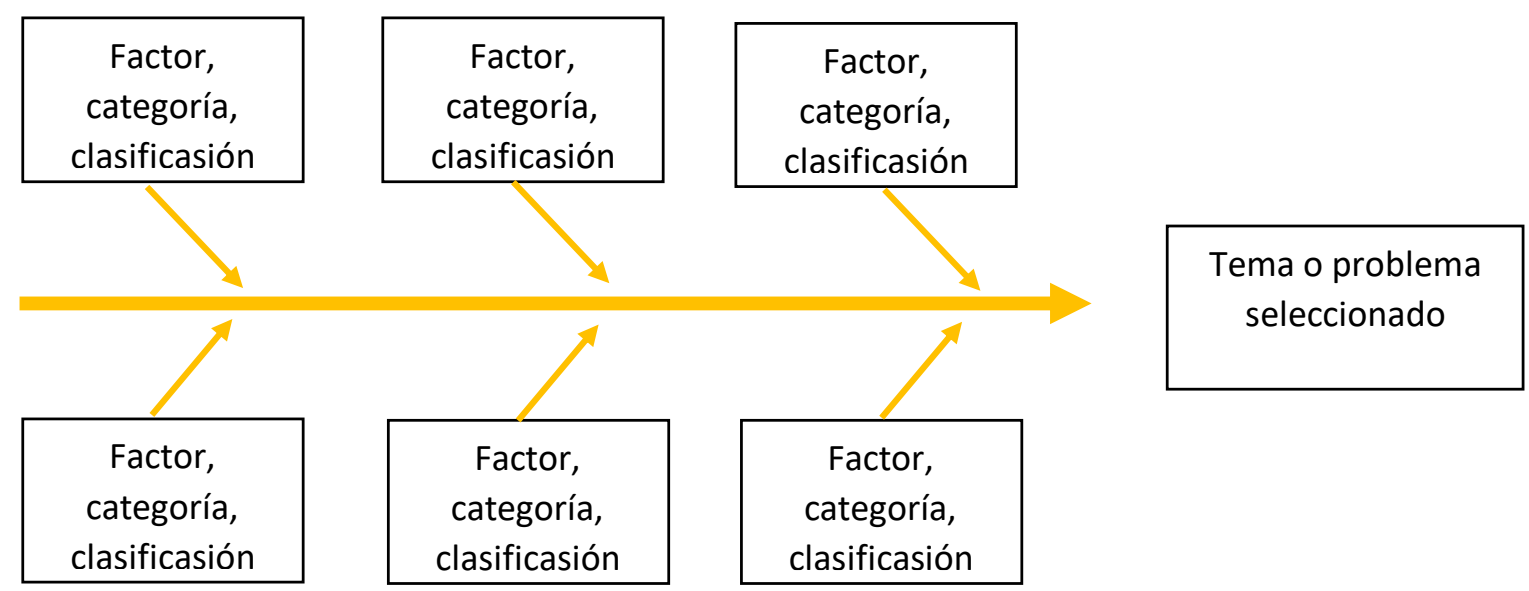

Fuente: Elaboración propia con información de Cantú (2011) 


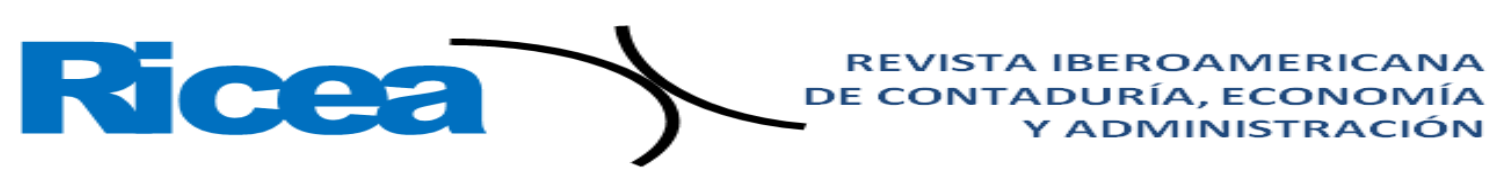

ISSN= $2007-9907$

Figura 3. Elemento

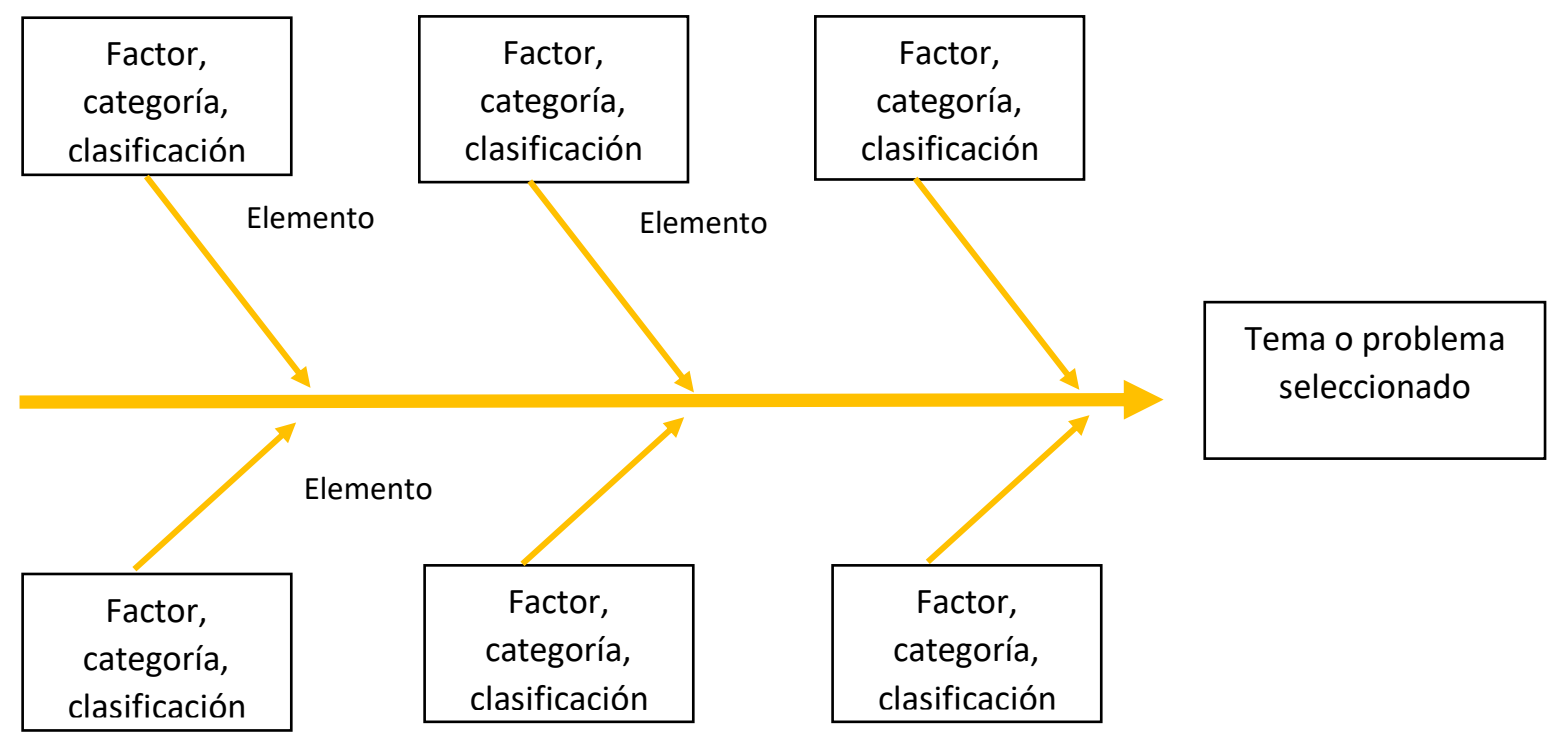

Fuente: Elaboración propia con información de Cantú (2011)

Como queda patente, la relación que existe entre los factores causales y el problema o tema seleccionado queda expresado mediante una gráfica que se puede interpretar de dos maneras: la inmediata es la relación existente entre el tema o problema seleccionado y las categorías o factores como causas potenciales generadoras de dicho tema o problema; la otra manera es la relación constituyente de los elementos que por su afinidad con el título de la categoría son la propia categoría desglosada en sus componentes.

Es importante la efectividad y objetividad del diagrama de causa-efecto, ya que exhibe las relaciones entre un problema y sus posibles causas, pero además permite al grupo (si fuera el caso) en futuras sesiones adelantar escenarios de potenciales soluciones al problema propuesto. Esta herramienta metodológica concretiza ampliamente lo que Hernández et al. (2014) llaman sesiones en profundidad. El verdadero objetivo es obtener la información en cantidad y calidad suficientes para satisfacer las necesidades de la investigación, y esta herramienta permite la selección de fuentes cuya interacción funciona como estímulo para la producción de dicha información. 
Tabla 1. Escolaridad de los entrevistados

\begin{tabular}{|c|c|c|c|c|c|c|}
\hline Edad & Profesional & Técnico & Bachiller & Secundaria & Primaria & Total \\
\hline $20-29$ & & & 2 & 4 & 1 & 7 \\
\hline $30-39$ & 1 & 1 & 4 & 3 & 1 & 10 \\
\hline $40-49$ & 1 & 1 & 4 & 11 & 11 & 28 \\
\hline $50-59$ & & 2 & & 5 & 12 & 19 \\
\hline $60-69$ & & & & 1 & 8 & 9 \\
\hline $70-79$ & & & 1 & 1 & 6 & 8 \\
\hline $80-+$ & & & & & 4 & 4 \\
\hline
\end{tabular}

Fuente: Elaboración propia

El análisis de estos datos nos lleva a exponer las siguientes consideraciones: del total de encuestados, 34 son menores de 45 años y de estos solo $32 \%$ se dedica a la actividad agrícola. Otro dato interesante es que $91 \%$ de estos reporta un nivel académico de secundaria para arriba. Por otra parte, los encuestados mayores de 45 años suman la cantidad de 51 personas, de las cuales $62.7 \%$ se dedica a la agricultura, y de estos, $63 \%$ reporta un nivel de escolaridad de primaria. De esta información obtenemos argumentos numéricos para deducir que a mayor nivel académico mayor distanciamiento de las actividades agrícolas. Pero existe un eslabón más en esta cadena: a mayor edad, menor nivel académico, lo que también explica el porqué la mayoría de los campesinos de Sta. María Texcalac son mayores de 45 años.

Tabla 2. Escolaridad de los entrevistados que practican la agricultura como actividad principal

\begin{tabular}{|l|c|c|c|c|}
\hline \multirow{2}{*}{ Edad } & \multicolumn{2}{|c|}{$20-\mathbf{4 4}$} & \multicolumn{2}{c|}{ 45-+ } \\
\cline { 2 - 5 } & $\#$ & $\%$ & $\#$ & $\%$ \\
\hline Profesional & 0 & 0 & 0 & 0 \\
\hline Técnico & 2 & 18 & 1 & 3 \\
\hline Bachillerato & 4 & 36 & 1 & 3 \\
\hline Secundaria & 4 & 36 & 10 & 31 \\
\hline Primaria & 1 & 10 & 20 & 63 \\
\hline Total & 11 & 100 & 32 & 100 \\
\hline
\end{tabular}

Fuente: Elaboración propia 


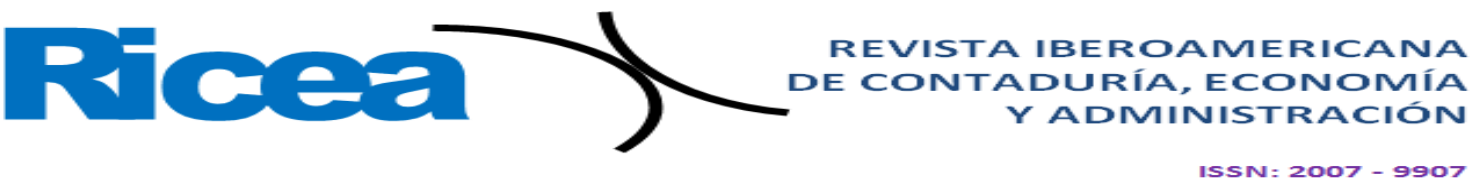

Un elemento que llama poderosamente la atención es que mientras más joven sea el entrevistado, la probabilidad de que su actividad principal sea la agricultura disminuye significativamente. Este dato es de suma importancia cuando se intenta realizar un diagnóstico sobre el futuro del campo en la población de Sta. María Texcalac.

La información obtenida es incuestionable, al menos esta encuesta así lo demuestra; en Sta. María Texcalac, Tlaxcala, las actividades agrícolas actualmente son practicadas en su mayoría por gente de edad, a los jóvenes parece no atraerles este sector productivo. La razón es porque el campo a nivel de pequeños propietarios o ejidatarios no es redituable, no proporciona ingresos para sostener una familia. Y por estas mismas razones las personas mayores incentivan en sus hijos otras actividades económicas diferentes a la agricultura.

Es imperativo no perder de vista que esta dinámica de cambio de actividad económica que se observa en la localidad se da en un escenario de proceso conurbatorio; el acceso que los habitantes de esta localidad tienen con la ciudad de Apizaco y la cercanía de medios de comunicación con otras ciudades como Huamantla ha facilitado y de alguna manera inducido a la búsqueda de otras actividades económicas que les proporcionen otro tipo de satisfactores.

Otro elemento importante que ha contribuido a facilitar la búsqueda de otras actividades económicas en detrimento de las del sector primario es el asentamiento de industrias de todos tamaños, las cuales empiezan a caracterizar parte de la panorámica de la localidad. Este proceso tiene dos consecuencias: por un lado, ofrece el acceso a los jóvenes a otro tipo de actividades económicas diferentes a las agropecuarias, y por otro, el campesino oferta sus terrenos para la construcción de infraestructura industrial o habitacional.

Tabla 3. Actividad económica

\begin{tabular}{|c|c|c|c|c|c|c|}
\hline \multirow{2}{*}{ Edad } & \multicolumn{2}{|c|}{ Agricultura } & \multicolumn{2}{c|}{ Otra } & \multicolumn{2}{c|}{ Total } \\
\cline { 2 - 7 } & $\#$ & $\%$ & $\#$ & $\%$ & $\#$ & $\%$ \\
\hline $\mathbf{2 0 - 4 4}$ & 11 & 32.4 & 23 & 67.6 & 34 & 100 \\
\hline $\mathbf{4 5 - 8 4}$ & 32 & 62.7 & 19 & 37.3 & 51 & 100 \\
\hline Total & 43 & 50.6 & 42 & 49.4 & 85 & 100 \\
\hline
\end{tabular}

Fuente: Elaboración propia 


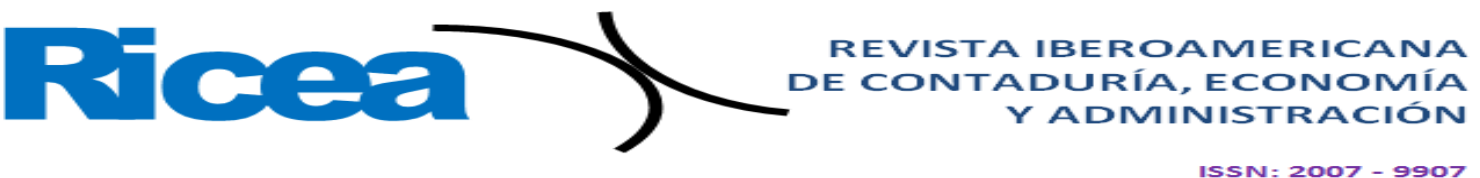

Como puede observarse en la tabla anterior, si bien la mitad de todos los entrevistados son campesinos, de los encuestados con más de 45 años, $63 \%$ son campesinos, mientras que solo $32 \%$ de los menores de 45 años dicen ser campesinos.

A los campesinos que dijeron tener una actividad económica complementaria (25\%), se les preguntó qué razones tuvieron para buscar esta. La respuesta fue unánime: $100 \%$ respondió: "Porque ya no alcanza lo que deja el campo".

Del total de los entrevistados, 56 practican la agricultura actualmente, ya sea como actividad principal (77\%) o como actividad complementaria $(23 \%)$. De estos datos resulta que actualmente 29 entrevistados no practican la agricultura ni como actividad principal ni como complementaria. Tomados estos últimos como elementos de análisis, encontramos lo siguiente: $41 \%$ practicaron la agricultura en otro tiempo. Las razones que tuvieron para abandonar esta actividad fueron:

$50 \%$ porque las actividades agrícolas se volvieron no redituables;

$25 \%$ porque vendió sus terrenos;

$17 \%$ por razones de enfermedad, y

$8 \%$ por edad.

Con la finalidad de conocer las perspectivas de los jóvenes en la comunidad, se les cuestionó lo siguiente: “¿Sus hijos se dedican actualmente a la agricultura?”. La respuesta a esta pregunta es reveladora y sintomática, quizá nos está dando un señalamiento que nos permite adelantar un escenario futuro de la actividad agrícola en Sta. María Texcalac, Tlaxcala. Esta pregunta fue aplicada a los encuestados que respondieron practicar la agricultura como actividad económica principal o como actividad económica complementaria y los resultados obtenidos muestran lo siguiente (véase tabla 4).

Tabla 4. Respuesta de los encuestados sobre si sus hijos se dedican o no a la agricultura

\begin{tabular}{|c|c|c|}
\hline \multirow{2}{*}{ Total } & \multicolumn{2}{|c|}{ Agricultura } \\
\cline { 2 - 3 } & $\#$ & $\%$ \\
\cline { 2 - 3 } & 56 & 100 \\
\hline Sí se dedica a la agricultura & 6 & 10.7 \\
\hline No se dedica a la agricultura & 50 & 89.3 \\
\hline
\end{tabular}

Fuente: Elaboración propia 


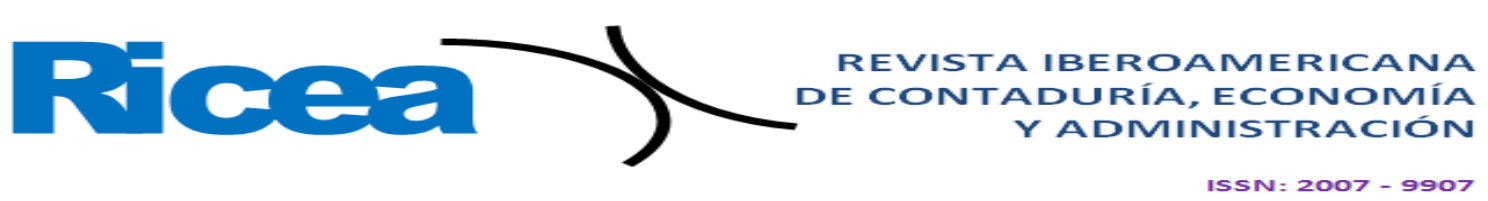

Los datos obtenidos en esta categoría apuntan a un abandono de la tradición campesina.

Al ser cuestionados sobre sus expectativas del campo y propuestas para evitar el abandono de las actividades agrícolas en la localidad, los habitantes de Sta. María Texcalac están convencidos de que el pequeño campesino, el ejidatario, no es atractivo para el Gobierno, que los buenos apoyos solo están destinados a los propietarios de grandes extensiones de terrenos, que los únicos campesinos que tienen futuro aquí mismo en la localidad son los que están "acaparando" terrenos mediante la compra de pequeñas propiedades.

Estos resultados son determinantes. El pequeño propietario de la tierra en esta localidad está convencido de que no reúne las características para ser tomado en cuenta como pasajero del carro de la modernidad. No es que no haya habido intentos de rescatar la actividad agrícola en la localidad, solo que los programas implementados, con toda su bondad, fracasaron por no tener un seguimiento adecuado.

Las experiencias tenidas de los participantes de estos programas no han sido halagüeñas; de alguna manera se han sentido engañados. Como lo manifiesta la Lic. Flores, expresidenta de comunidad, quien narra que se promovieron programas de ganado vacuno y granjas avícolas que tenían todo para triunfar, sin embargo, se cancelaron por cambio de autoridad.

También las asociaciones han sido un ensayo que generalmente, por falta de un liderazgo auténtico y desinteresado, no han llegado a feliz término. Los intentos particulares que los pequeños propietarios han hecho por conseguir apoyos oficiales no han tenido la efectividad esperada. Un testimonio de un campesino participante en la dinámica de grupo afirmó textualmente: "Si yo tuviera las garantías que me exigen para concederme un préstamo para la adquisición de un pequeño tractor, me lo compraba de contado sin tener que estarles pidiendo".

Todo se conjuga para que el Gobierno aparezca como el único responsable de la situación por la que atraviesa la actividad agrícola en localidades como la que estudiamos. Sin embargo, no es así: los campesinos no solo esperan créditos o subsidios, ven un mercado inundado de productos agrícolas frente a los cuales, aunque saben de su calidad dudosa, no pueden ofrecer competencia por el precio en que se ofertan. Pero aun así están convencidos de que una decisión política seria para rescatar el campo es posible. 
Un testimonio que parece ir en contra de la opinión general lo expresa el señor José Quintero, uno de nuestros informantes claves, quien afirma con convicción que "un día el Gobierno se va a dar cuenta de que el pequeño campesino es muy importante y va a hacer algo para que vuelva a producir sin tener que comprar al extranjero".

De hecho, por apoyos del Gobierno, $79 \%$ de los entrevistados están dando a entender programas que tengan por objetivo un verdadero rescate de las actividades agrícolas a nivel de pequeño propietario, de minifundistas; programas que tengan por objetivo hacer atractivo al campo, que permitan dar una rentabilidad a la actividad agrícola que pueda mantenerse por sí sola y así evitar venderlo. A medida que los conocimientos de la situación agrícola de estas localidades se incrementen existirá una mayor capacidad para detectar, diseñar o descubrir las acciones o programas más apropiados a los que hacen referencia los entrevistados, y sobre todo crear condiciones que los vuelvan atractivos para ser adoptados como propios por los protagonistas, de tal manera que su perdurabilidad sea la primer garantía de su efectividad.

\section{Entrevista con personajes clave}

La tabla 5 muestra las características de las personas elegidas como informante claves para realizar entrevistas con la metodología de entrevistas en profundidad. 
Tabla 5. Perfil de informantes clave

\begin{tabular}{|l|c|c|c|c|c|}
\hline \multirow{2}{*}{ Nombres } & \multirow{2}{*}{ Edad } & \multicolumn{3}{c|}{ Cargos Ocupados } & \multirow{2}{*}{ Actividad Económica } \\
\cline { 3 - 5 } & & Presidente & Comisariado & Fiscal & Docencia \\
\hline $\begin{array}{l}\text { Lic. Yolanda Flores } \\
\text { Lezama }\end{array}$ & 45 & $\mathrm{X}$ & & & Comercio/Campesino \\
\hline Sr. José Quintero López & 94 & $\mathrm{X}$ & $\mathrm{X}$ & $\mathrm{X}$ & Campesino \\
\hline $\begin{array}{l}\text { Sr. Juan Acosta Quiroz } \\
\text { Prof. Ramón Flores } \\
\text { García }\end{array}$ & 63 & $\mathrm{X}$ & $\mathrm{X}$ & $\mathrm{X}$ & Docencia \\
\hline $\begin{array}{l}\text { Sr. Arturo Hernández } \\
\text { Acosta }\end{array}$ & 61 & $\mathrm{X}$ & & & Campesino \\
\hline $\begin{array}{l}\text { Sr. Ponciano Duran } \\
\text { Toscuento }\end{array}$ & 55 & $\mathrm{X}$ & $\mathrm{X}$ & & Campesino \\
\hline
\end{tabular}

Fuente: Elaboración propia

Como puede observarse, es innegable el valor de la información de personas con este perfil, toda su vida han radicado en la población de Santa María Texcalac, y el hecho de haber ocupado cargos de autoridad en la localidad es indicio del conocimiento que los habitantes de esta comunidad tienen de ellos y viceversa.

Los resultados más importantes obtenidos de estas entrevistas a profundidad se consignan en el siguiente cuadro. 


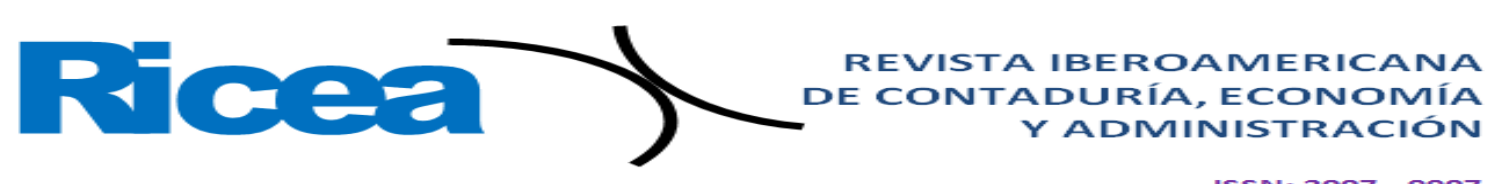

Tabla 6. Información obtenida de entrevistas con informantes clave

\begin{tabular}{|c|c|c|}
\hline \multirow[b]{2}{*}{ Opinión } & \multicolumn{2}{|c|}{$\begin{array}{l}\text { Tabla de } \\
\text { aparición }\end{array}$} \\
\hline & \# & $\%$ \\
\hline $\begin{array}{l}\text { El campo no es redituable. Se le invierte demasiado y } \\
\text { no se recupera ni lo invertido. No se puede vivir del } \\
\text { campo. }\end{array}$ & 6 & 100 \\
\hline A los jóvenes no les interesa el campo. & 6 & 100 \\
\hline El futuro de la actividad agrícola es el abandono. & 6 & 100 \\
\hline Existe acaparamiento de tierras. & 6 & 100 \\
\hline $\begin{array}{l}\text { No existen apoyos efectivos del Gobierno al pequeño } \\
\text { campesino. }\end{array}$ & 6 & 100 \\
\hline Los actuales campesinos son gente de edad avanzada. & 4 & 66 \\
\hline $\begin{array}{l}\text { El campesino vende o deja su pequeña propiedad y } \\
\text { emigra. }\end{array}$ & 3 & 50 \\
\hline Gente ajena a la comunidad está comprando terrenos. & 3 & 50 \\
\hline
\end{tabular}

Fuente: Elaboración propia

Es necesario mencionar que estas entrevistas con "informantes clave" fueron realizadas de manera individual y en diferentes fechas, por lo tanto, no existió la posibilidad de comunicación entre ellos que facilitara el comentario y el acuerdo de respuestas, así como tampoco la imitación en las mismas. Esta aclaración es pertinente porque el análisis de la información obtenida pareciera un acuerdo de opiniones. En cinco categorías nuestros entrevistados coinciden en $100 \%$. Ya el análisis de los resultados de la encuesta lo mencionaba que "el campo no es redituable". Esta comunión de resultados nos lleva a la conclusión de que una de las razones por las cuales la actividad agrícola en el pueblo de Sta. María Texcalac, Tlaxcala, está siendo abandonada es precisamente porque "no se puede vivir del campo". Esta razón se complementa con la opinión, también externada por la totalidad de los informantes, de que "el futuro de la actividad agrícola en los parvifundistas es el abandono"; la relación conceptual entre estas dos categorías es evidente.

La información obtenida mediante estas entrevistas confirman los resultados obtenidos mediante la aplicación de cuestionarios. El total de nuestros informantes clave coincide en que "no existen apoyos efectivos del Gobierno al pequeño campesino"; no solo 


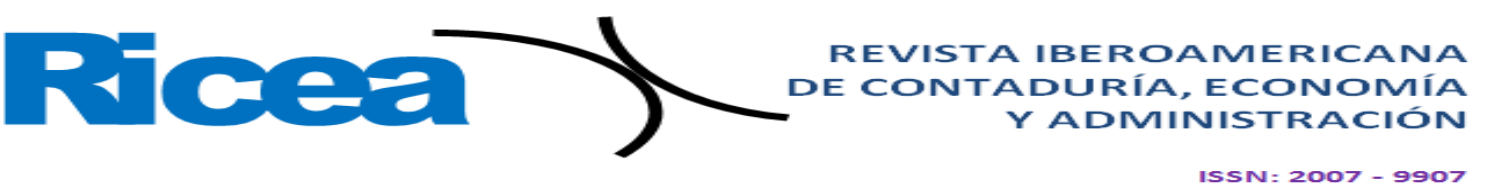

se refieren a apoyos monetarios, sino a verdaderos programas de rescate de las actividades agrícolas destinados a pequeños propietarios y ejidatarios.

Otras dos opiniones que guardan una relación de causalidad con el abandono de las actividades agrícolas son que "los campesinos actuales son gente de edad avanzada" y que “a los jóvenes no les interesa el campo". Estos resultados también ya los encontramos en el análisis de las otras técnicas utilizadas en la investigación de campo.

\section{Resultados del taller Ishikawa}

Los resultados obtenidos de la aplicación de este método para obtener información directa, objetiva, retroalimentada y suficiente para alcanzar las metas ya especificadas cumplieron más allá de las expectativas calculadas. Estamos seguros de que el éxito obtenido estuvo fincado en el respeto a la metodología que exige de antemano esta herramienta (Cantú, 2011).

Uno de los factores que jugó un papel decisivo en la riqueza de la información recopilada fue la selección que se llevó a cabo de los participantes en esta dinámica de grupo. El grupo estuvo integrado por las siguientes personas:

Sr. Maximino F. García, 60 años, recién jubilado de una empresa de servicios del sector salud. Proviene de una familia eminentemente campesina. Actualmente combina actividades agrícolas con otras actividades económicas. Ocupó el segundo cargo en importancia en la administración del templo de la localidad.

Sr. Horacio Picazo Sánchez, 38 años. Su actividad económica actual es el comercio de carne. Posee terrenos dedicados a las actividades agrícolas que renta a otras personas.

Sr. Virgilio Huerta Sánchez, 71 años, jubilado de una empresa de servicios del sector eléctrico. Proviene de una familia de campesinos. Posee terrenos agrícolas que son trabajados por familiares.

Sra. Ma. Esther Rodríguez, 58 años. Ama de casa, ayuda a su esposo en las actividades agrícolas. Tiene otras actividades económicas, minicomercio de golosinas, para apoyar el ingreso familiar. 


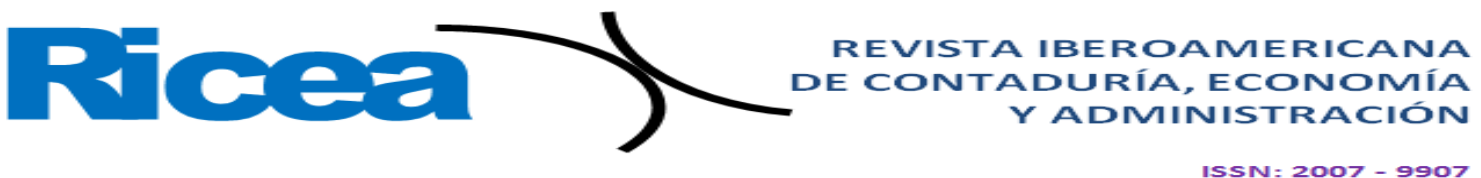

Sr. Roberto Flores G., 55 años. Dedicado al campo por tradición familiar. Realiza otras actividades económicas que consisten en una pequeña miscelánea que le proporcionan el complemento del ingreso en el hogar

El resultado de la lluvia de ideas que se produjo acerca de la situación de las actividades agrícolas en Sta. María Texcalac, Tlaxcala, arroja el siguiente diagrama de causaefecto (ver figura 4).

Figura 4. Diagrama de Ishikawa

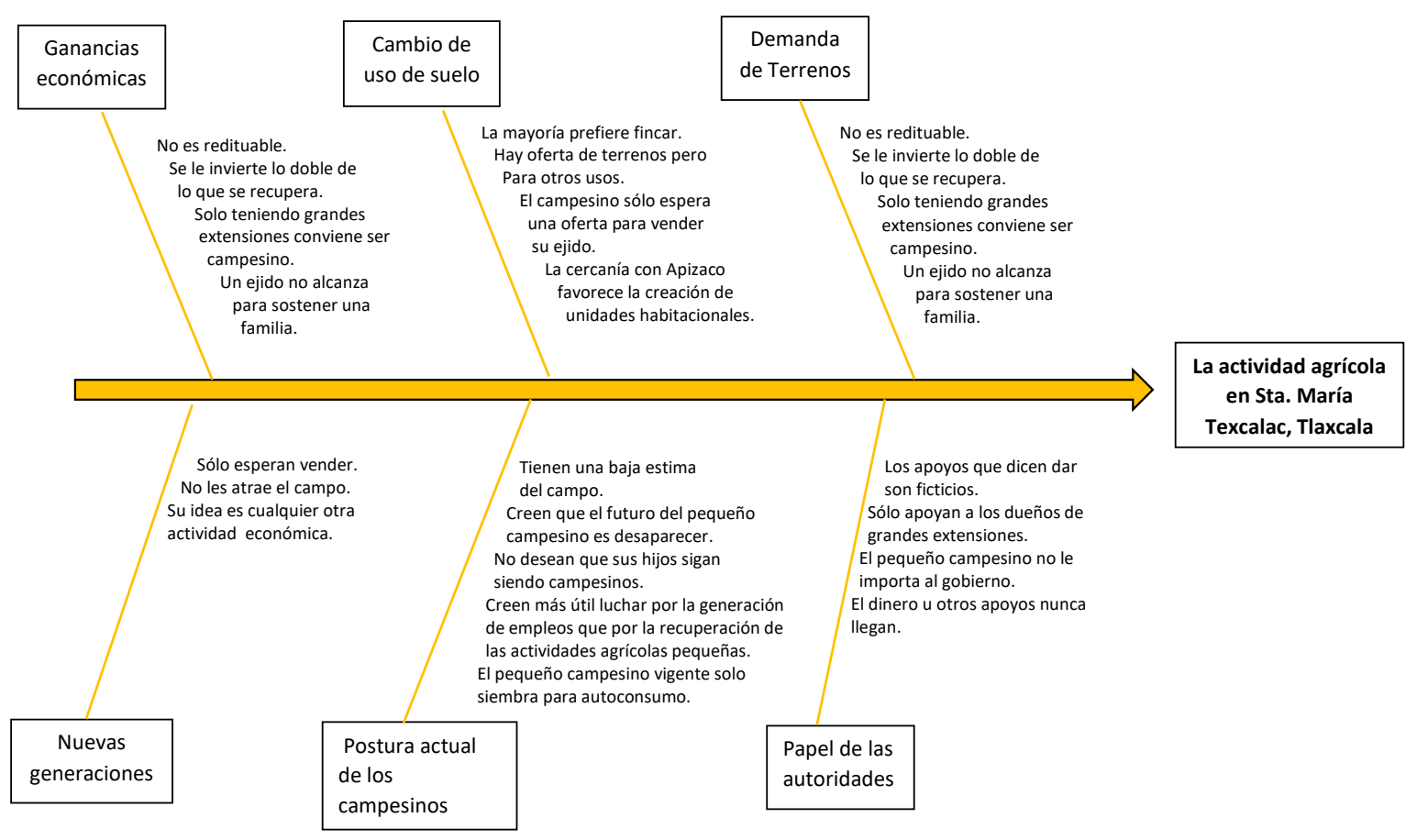

Fuente: Elaboración propia

Este diagrama de causa-efecto traza una ruta muy similar a la genereda por los resultados obtenidos mediante las otras herramientas de la investigación de campo. Existen elementos que saltan a la vista y que no pasaron desapercibidos para el moderador de esta dinámica. Todos los integrantes coincidieron, y no perdieron oportunidad de reiterar, en que el campo "a baja escala", o sea, como práctica de minifundio o pequeño campesino o ejidatario, es incosteable porque el gasto económico que exigen no se recupera con el producto obtenido. En este punto fueron tajantes: el ejido o la pequeña propiedad no es redituable. Este resultado es reiterativo en todo el proceso de investigación. Un análisis de la información obtenida mediante este método conduce a la confirmación de resultados de los 


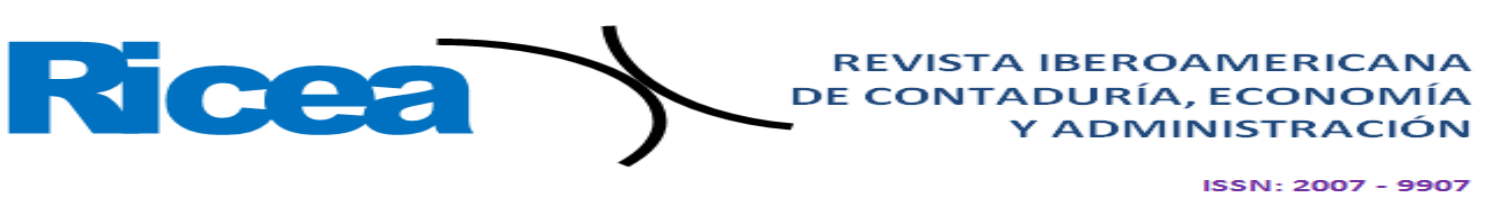

otros métodos utilizados en la investigación de campo. Los resultados coinciden y se concentran básicamente en las siguientes categorías:

- $\quad$ La actividad agrícola a nivel ejido o pequeña propiedad no es redituable.

- Los campesinos actuales en la comunidad de Sta. María Texcalac son gente mayor.

- $\quad$ A los jóvenes parece no interesarles las labores del campo.

- Los programas del Gobierno no hacen atractivas las actividades agrícolas.

Todas las otras opiniones y temas de sus intervenciones parecían girar o tener como sustrato estos tópicos. Leyendo entre líneas podría interpretarse que estas temáticas son las generadoras o las causales de la situación crítica de abandono que están sufriendo las actividades agrícolas en Sta. María Texcalac.

\section{Conclusiones}

En esta localidad encontramos una estrecha relación entre el visible abandono paulatino de las actividades agrícolas y el implantamiento de las políticas neoliberales. Esta relación se refleja, sobre todo, en el clamor de los pequeños propietarios a razón de que los apoyos gubernamentales solo están al alcance de los grandes productores agrícolas. La actividad agrícola en esta localidad está disminuyendo progresivamente no solo por los efectos del proceso de conurbación, sino también como una consecuencia de las políticas liberales que privilegian las importaciones de alimentos libres de aranceles. Este tipo de toma de decisiones empuja definitivamente al pequeño campesino a abandonar su actividad agrícola y a buscar otra actividad económica que le permita la supervivencia.

La investigación de campo fue determinante para alcanzar las metas. Las herramientas elegidas tuvieron por objeto confirmar la información obtenida, funcionaron a la manera de test-retest y los sujetos de investigación permitieron tener una cobertura que alcanzara una representatividad de la población de tal manera que no existiera la menor duda al respecto. Ahora bien, este trabajo conduce a la conclusión de que los factores causales del abandono de las actividades agrícolas en Sta. María Texcalac son tanto endógenos como exógenos; entre los primeros tenemos que "a los jóvenes no les interesa las actividades agrícolas" y que "70 \% de los campesinos en Sta. María Texcalac son gente de edad avanzada (mayores de 45 años)"; entre los segundos encontramos a las "políticas económicas", que "el campo a nivel de ejido o pequeña propiedad no es redituable", "la demanda de tierras para 


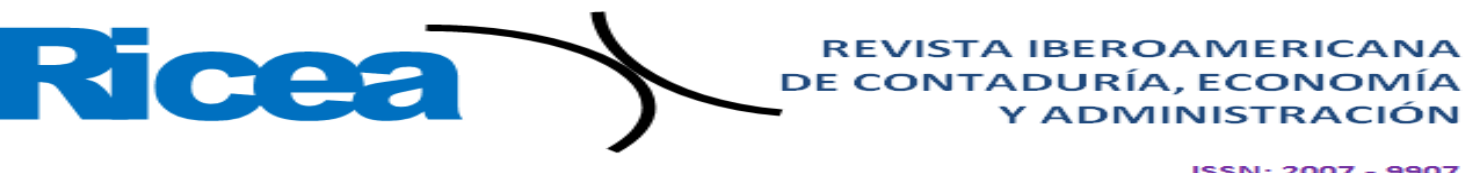

infraestructura industrial y de vivienda", "la insuficiencia de los apoyos gubernamentales" y "los fenómenos climatológicos".

Quizá uno de los signos que refleja abiertamente este proceso conurbatorio sea el crecimiento desmesurado del sector transporte en la comunidad. El tiempo en el que se ha incrementado no concuerda ni con el incremento poblacional ni con el desarrollo de otros rubros dentro de la propia comunidad, por ejemplo, el sector salud, la infraestructura que tenga por objeto el fomento de la cultura y entretenimiento, además de otros sectores que parecen estancados desde hace mucho años, sin embargo, el flujo diario de la población hacia la mancha urbana durante todo el día da cuenta de que esta ciudad se ha convertido en un centro de actividades que ya empieza a ser considerado como un anexo periférico.

Los resultados de cada una de las actividades de investigación fueron verdaderamente enriquecedores. La información recopilada nos deja en posición de entender la problemática por la que pasa el pequeño agricultor que se niega a morir pero que, a pesar de sus esfuerzos, ve que el fin de toda una tradición familiar está próximo.

Concluimos con desencanto que las alternativas que tiene la agricultura en una sociedad con estas características para subsistir no son tan factibles como uno quisiera, y no lo son porque tanto los factores endógenos como exógenos no son propicios. Por una parte, la actitud del campesino de la localidad es de resignación, ya no tiene la intencionalidad de reactivar las actividades agrícolas; y por otra, la política gubernamental que prefiere comprar alimentos que producirlos se suma para frenar cualquier intento de reactivación en este tipo de actividades. El pequeño campesino está inerme ante la política económica neoliberal. Solo le resta seguir siendo campesino por amor a la tierra. Esto se muestra en los diferentes testimonios, tanto en las entrevistas, en las dinámicas, como en la encuesta.

Aunado a lo anterior, se encuentra que el pequeño productor está en total desventaja para poder llegar a ser competitivo dentro del mercado. El pequeño campesino de Santa María Texcalac prefiere utilizar el maíz que le queda, después de satisfacer sus necesidades de autoconsumo, como alimento de animales antes que "malbaratarlo", porque el precio de mercado es irrisorio; cuando debería pagarse este maíz a un precio especial, ya que es producto de un trabajo verdaderamente artesanal. El desencanto de los campesinos es tal que el Sr. Roberto Flores García, participante del taller, expresaba lo siguiente: "Si al Gobierno no le interesamos, debería de una vez implantar en nuestro pueblo industrias que le den trabajo a nuestros hijos y olvidarnos del campo". 


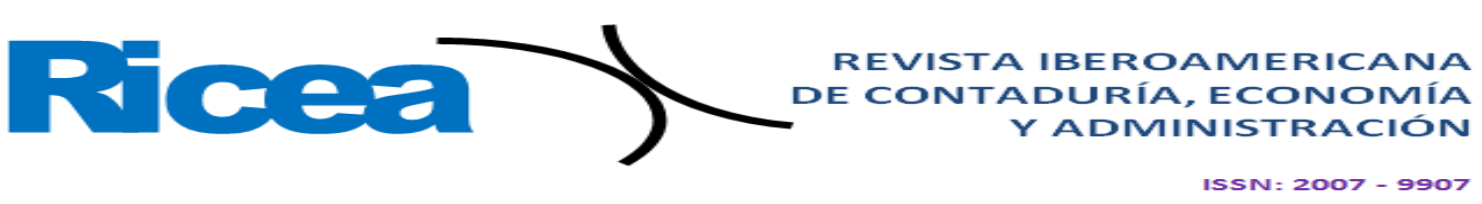

Por otra parte, debe mencionarse que los campesinos de la localidad no están en una situación de hambre o desnutrición, de ahí que su postura no es el reclamo desesperado de una circunstancia extrema. Los campesinos de esta localidad han aprendido a no vivir exclusivamente del campo, ya sea por la adopción de una actividad complementaria o porque otros miembros de la familia aportan recurso económico. Sin embargo, el fantasma del hambre y la desnutrición no debe descartarse y debe ser considerado como una posibilidad futura. Por lo que, bajo estas consideraciones, puede afirmarse que los tiempos son apropiados para tomar medidas preventivas que impidan a comunidades rurales que se encuentran en estos procesos de urbanización o conurbación perder de vista los riesgos que conlleva el abandono de las actividades agrícolas, y la ventaja que implica el conservar dichas actividades para garantizar una seguridad y sobre todo soberanía alimentaria.

Los elementos que desfavorecen esta convivencia son el desorden y la falta de planificación con los que se instalan pequeñas, medianas y grandes industrias que tientan al campesino a vender sus terrenos para ser utilizados en la creación de esta infraestructura, la cual, a su vez, influye principalmente en la juventud para emprender una búsqueda de actividades económicas diferentes a las de sus padres. Otra consecuencia de este asentamiento de industrias es la ruptura del equilibrio ecológico en la región, específicamente en la localidad de Santa María Texcalac, donde sus afluentes son testigos de la contaminación que produce dicha infraestructura. Un proyecto que tenga en cuenta estos elementos negativos seguramente tendrá mayores probabilidades de éxito, pero sobre todo recuperará paulatinamente el sentido de localidad en los habitantes de comunidades que, como el caso de Santa María Texcalac, están viviendo un proceso de conurbación que no es fácil de digerir ni asimilar, ya que implica un profundo cambio en todos los órdenes de la vida de los habitantes de estas localidades. 


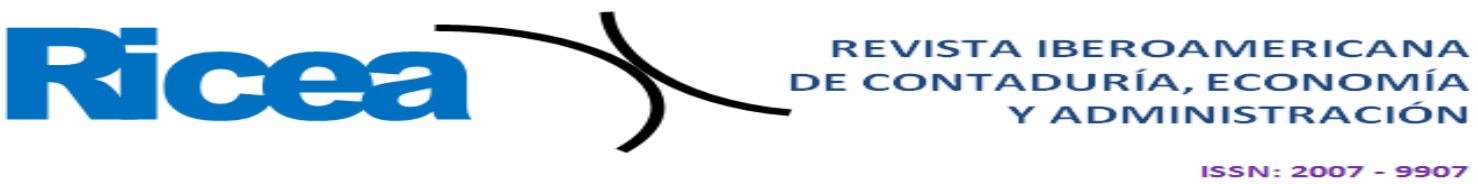

\section{Referencias}

Bracamonte, R. (2015). La observación participante como técnica de recolección de información de la investigación etnográfica. Revista ARJÉ, 9(17).

Comisión Económica para América Latina y el Caribe [Cepal]. (2010). Crecimiento urbano de América Latina. San José, Costa Rica: Comisión Económica para América Latina y el Caribe.

Cantú, H. (2011). Desarrollo de una cultura de calidad (4.a ed.). Ciudad de México, México: McGraw-Hill.

Fondo de Población de las Naciones Unidas [Fnuap]. (2018). ONU: Dos terceras partes de la población vivirán en centros urbanos para 2050. Fondo de población de las Naciones Unidas.

Hernández, R., Fernández, C. y Baptista, M. (2014). Metodología de la Investigación (6. ${ }^{\text {a }}$ ed.). Ciudad de México, México: McGraw-Hill.

Instituto Nacional de Estadística y Geografía [Inegi]. (1980). X Censo General de Población y Vivienda. Iter. Principales resultados por localidad. México: Instituto Nacional de Estadística y Geografía.

Instituto Nacional de Estadística y Geografía [Inegi]. (1990). XI Censo General de Población y Vivienda. Iter. Principales resultados por localidad. México: Instituto Nacional de Estadística y Geografía.

Instituto Nacional de Estadística y Geografía [Inegi]. (2000). XII Censo General de Población y Vivienda. Iter. Principales resultados por localidad. México: Instituto Nacional de Estadística y Geografía.

Instituto Nacional de Estadística y Geografía [Inegi]. (2010). XIII. Censo de Población y Vivienda. Iter. Principales resultados por localidad. México: Instituto Nacional de Estadística y Geografía.

Kautsky, Karl. (2002). La cuestión agraria (2. a ed.). México: Siglo XXI Editores.

Moreno, O. (2004). A propósito de los procesos de conurbación en el sur de la metrópoli bogotana. Revista Bitácora Urbano Territorial, 1(8).

Naredo, J. (2000). Sobre la insostenibilidad de las actuales conurbaciones y el modo de paliarla. Gaceta Ecológica, (55).

Naredo, M. (2003). El metabolismo económico de la conurbación madrileña 1984 - 2001. Economía Industrial, (351) 


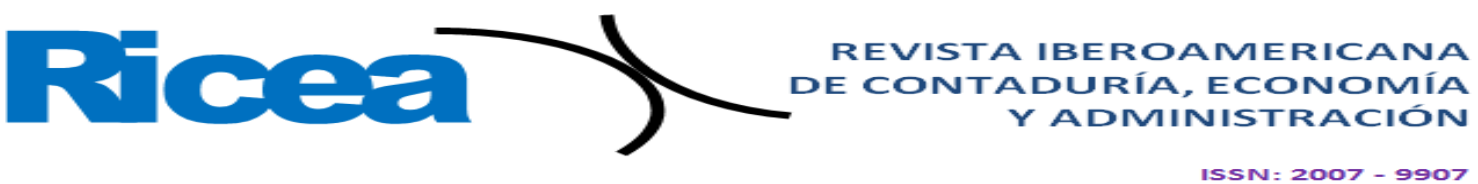

Navarro, H. (2005). Transformaciones de los territorios periurbanos y sus agriculturas: El uso de recursos de interés público en el Valle de México. En Ávila, H. (comp.), Lo urbano-rural, ¿nuevas expresiones territoriales? Cuernava, México: Centro Regional de Investigaciones Multidisciplinarias. UNAM.

Pellicer, I., Vivas, P. y Rojas, J. (2013). La observación participante y la deriva: dos técnicas móviles para el análisis de la ciudad contemporánea. El caso de Barcelona. EURE, 39(116).

Quispe, A. (1999). El diseño de cuestionarios, una forma práctica de hacer preguntas con un propósito (1. ${ }^{\mathrm{a}}$ ed.). Tlaxcala, México: Colegio de Postgraduados. Universidad Autónoma de Tlaxcala.

Valladares, L. (coord.) (2008). Metropolización, conurbación y dispersión. Los municipios del departamento de Guatemala (1986 - 2007). Guatemala, Guatemala: Universidad de San Carlos. 


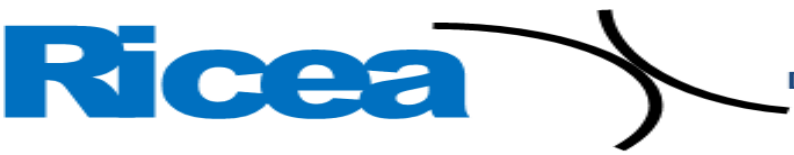

REVISTA IBEROAMERICANA DE CONTADURÍA, ECONOMIIA

ISSN $=2007-9907$

\begin{tabular}{|l|l|}
\hline Rol de Contribución & Autor (es) \\
\hline Conceptualización & José Luis Carmona Silva \\
\hline Metodología & José Luis Carmona Silva \\
\hline Software & No aplica \\
\hline Validación & José Luis Carmona Silva \\
\hline Análisis Formal & José Luis Carmona Silva \\
\hline Investigación & José Luis Carmona Silva \\
\hline Recursos & $\begin{array}{l}\text { José Luis Carmona Silva. Principal José Aurelio Cruz de los } \\
\text { Ángeles. Apoyo Lizbeth Sánchez Flores. Apoyo }\end{array}$ \\
\hline Curación de datos & José Luis Carmona Silva \\
\hline $\begin{array}{l}\text { Escritura - Preparación del } \\
\text { borrador original }\end{array}$ & José Luis Carmona Silva \\
\hline $\begin{array}{l}\text { Escritura - Revisión y } \\
\text { edición }\end{array}$ & $\begin{array}{l}\text { José Luis Carmona Silva José Aurelio Cruz de los Ángeles. } \\
\text { Apoyo Lizbeth Sánchez Flores. Apoyo }\end{array}$ \\
\hline Visualización & $\begin{array}{l}\text { José Luis Carmona Silva José Aurelio Cruz de los Ángeles. } \\
\text { Apoyo Lizbeth Sánchez Flores. Apoyo }\end{array}$ \\
\hline Proyectos & José Luis Carmona Silva \\
\hline Adquisición de fondos & José Luis Carmona Silva José Aurelio Cruz de los Ángeles. \\
\hline Apoyo Lizbeth Sánchez Flores. Apoyo
\end{tabular}

